\title{
Percutaneous Peripheral Nerve Stimulation (Neuromodulation) for Postoperative Pain: A Randomized, Sham-controlled Pilot Study
}

Brian M Ilfeld et al. Anesthesiology 2021; 135:95-110

$\mathrm{P}$

ostoperative Schmerztherapie ist ein integraler Bestandteil der perioperativen Aufgaben unseres Faches. Trotz Verwendung einer Vielfalt von pharmakologischen und interventionellen Methoden ist die postoperative Analgesie oft unzureichend.

Patient*innenkontrollierte Analgesie hat sich in vielen Institutionen etabliert.

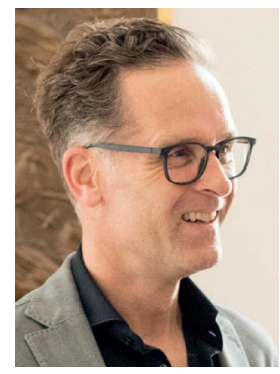

Von

PD Dr.

Gerhard Fritsch

AUVA, UKH Salzburg

Aufgrund fehlender struktureller Vor-

aussetzungen bzw. ihrer Wirkungs- und Nebenwirkungsprofile ist sie aber oft immer noch nicht verfügbar oder indiziert.

Die Chronifizierung von postoperativen Schmerzen ist mit 30 bis 50 Prozent ein hochrelevantes Problem, das oft noch mit Opioid-Abhängigkeit vergesellschaftet ist.

Kontinuierliche perineurale Schmerztherapie unter Verwendung von Kathetern kann relativ nebenwirkungsarm und effektiv postoperative Schmerzen lindern, birgt aber immer das Risiko von pharmakologischen Komplikationen und lokalen Infektionen.

Brian Ilfeld beschäftigt sich schon seit Jahren mit nichtpharmakologischen Methoden zur Ausschaltung akuter und chronischer Schmerzen. Dazu verwendet er, vergleichbar mit spinalen Stimulationssonden, elektrische Spannungen mit geringer Intensität, die mittels eigener Elektroden ins Gewebe eingebracht werden und mit einem externen Stimulationsgenerator verbunden sind. 2018 hat die FDA eine solche Sonde zugelassen.

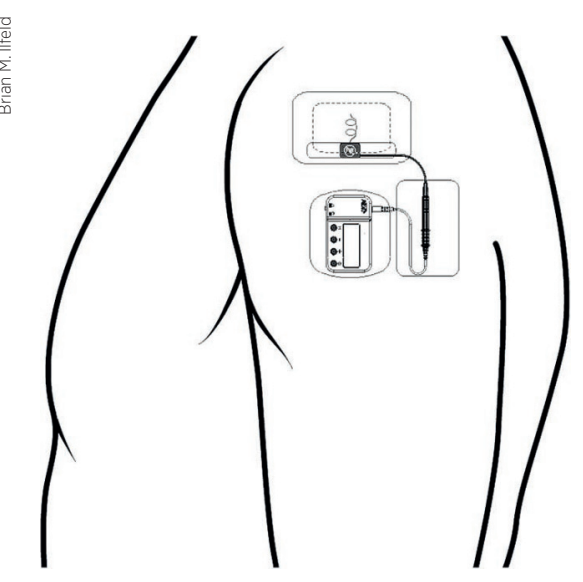

Body-worn percutaneous peripheral nerve stimulation system used de elektrisch stimuliert, in der Kontrollgruppe wurde eine sogenannte Sham-Behandlung durchgeführt. Das heißt, dass zwar der Stimulationsgenerator an die Sonde angebracht, aber nicht stimuliert wurde.

In der vorliegenden Studie wurde die Effektivität dieser Methode geprüft. Dazu wurden insgesamt 66 Patienten und Patientinnen, die sich fünf verschiedenen orthopädischen Eingriffen unterzogen, randomisiert und zwei Gruppen zugeordnet. In der Behandlungsgruppe wurde die zuvor eingebrachte Sonaber nicht stimuliert wurde.
Als zu untersuchende Endpunkte definierte man einerseits den Opioid-Verbrauch und andererseits die Schmerzintensität zu verschiedenen Zeitpunkten. Weiters wurden mittels Fragebogen Lebensqualität und Einschränkung durch Schmerzen abgefragt. Die Teilnehmer*innen der Studie durften je nach Intensität ihrer Schmerzen zusätzlich orale Opioide einnehmen. Die Eingriffe waren allesamt ambulant, die Stimulationssonde und der dazugehörige Stimulationsgenerator wurden den Patient*innen nach vorheriger Einschulung mitgegeben. Am 14. postoperativen Tag wurde das System entfernt.

Die Ergebnisse waren in mehrerlei Hinsicht eindrucksvoll. Sowohl der Opioid-Konsum als auch die durchschnittlichen Schmerzwerte waren in der Behandlungsgruppe gegenüber der Kontrollgruppe signifikant niedriger. Über die ersten sieben Tage war die kumulative Opioid-Dosis in Morphinäquivalenten in der Stimulationsgruppe fast um das Zehnfache niedriger. Die durchschnittliche Schmerzintensität war um mehr als die Hälfte reduziert. Bezüglich der schmerzbedingten Einschränkungen ergaben sich kaum Unterschiede zwischen den Gruppen, wobei auffallend war, dass in der Sham-Gruppe nach wie vor sechs Patient*innen Opioide benötigten.

In der sehr detaillierten Darstellung der Ergebnisse wurden auch Komplikationen dokumentiert. Bei der Entfernung des Systems ergaben sich zwei Sondenbrüche, bei einem Patienten kam es zu einer Rötung der Eintrittsstelle der Stimulationssonde. Bei einem der verwendeten Geräte trat ein technischer Defekt auf, der unmittelbar behoben werden konnte. Die in einer früheren Studie von Ilfeld aufgetretenen unangenehmen Muskelzuckungen traten in abgeschwächter Form bei neun Patient*innen auf. Über die genaue Charakteristik der verwendeten Stromstärke und der Impulsdauer lässt die Studie Leser*innen im Unklaren. Hier beruft man sich auf das Recht des geistigen Eigentums.

FAZIT für die Praxis: Hier bahnt sich eine Revolution der regionalen postoperativen Schmerztherapie an. Im Vergleich zu bereits veröffentlichten Daten scheint sich die Praxistauglichkeit solcher Systeme deutlich verbessert zu haben. Es ist mehr als nur interessant, Patient*innen eine nebenwirkungsarme Alternative zu pharmakologischen Methoden - mit oder ohne Verwendung von Regionalanästhesie - zu bieten sowie zusätzlich noch die Möglichkeit, die Intensität der Behandlung selbst zu regeln. Eine große Untersuchung mit 250 Teilnehmer*innen ist bereits in Planung. 